\title{
El estrés laboral y su relación con el desempeño de los promotores de servicio de las agencias del distrito de Magdalena del Mar, en una institución financiera, 2019 - 2020
}

\author{
Elizabeth del Carmen Velásquez Fuentes \\ elizabethdelcarmenvelasquezfue@gmail.com
}

ORCID: 0000-0003-2235-1904

\section{RESUMEN}

El objetivo de investigación ha sido determinar la relación que existe entre el estrés laboral con el desempeño de los promotores de servicio de las agencias del distrito de Magdalena, en una Institución Financiera. La hipótesis general ha comprobado lo siguiente el estrés laboral se relaciona significativamente con el desempeño de los promotores de servicio de las agencias del distrito de Magdalena, en una Institución Financiera. El diseño de investigación ha sido de tipo descriptivo - correlacional, de tipo cuantitativo. Se emplearon dos instrumentos de investigación, uno orientado a los promotores de las agencias y el segundo a los jefes. Para determinar la confiabilidad de los ítems de los instrumentos, se aplicó el coeficiente de Alfa de Cronbach, para la variable estrés se obtuvo un nivel de confianza de 0.75 , la prueba piloto fue aplicada a una muestra de 15 colaboradores de la institución financiera en estudio. De igual manera, el instrumento que ha servido para analizar el desempeño laboral se ha obtenido un nivel de confianza de 0.82 lo cual es significativo para la investigación.

La comprobación de la hipótesis ha quedado comprobada y demostrada a través del Modelo Estadístico utilizado, se ha concluido que el estrés laboral se relaciona significativamente con el desempeño laboral de los promotores de Servicio de las Agencias del Distrito de Magdalena, en una Institución Financiera, determinándose una Chi cuadrado de Pearson de 0.576. Se comprobó la existencia de problemas de estrés en los trabajadores, identificándose 4 casos de estrés; pudiéndose afirmar que ello repercute de manera negativa en el desempeño laboral de los promotores, concluyéndose que las consecuencias afectan la productividad en cada una de las agencias donde laboran los promotores.

Palabras clave: estrés laboral; desempeño; burnout; agotamiento emocional; efecto fisiológico 


\title{
Labor Stress and its relationship with the performance of service promoters of the Magdalena del Mar district agencies, in a financial institution, $2019-2020$
}

\begin{abstract}
$\mathrm{X}$ The research objective has been to determine the relationship that exists between work stress and the performance of service promoters of the Magdalena district agencies, in a Financial Institution. The general hypothesis has verified the following: work stress is significantly related to the performance of the service promoters of the Magdalena district agencies, in a Financial Institution. The research design has been descriptive correlational, quantitative. Two research instruments were used, one aimed at promoters of the agencies and the second at heads. To determine the reliability of the items of the instruments, the Cronbach's alpha coefficient was applied, for the stress variable a confidence level of 0.75 was obtained, the pilot test was applied to a sample of 15 collaborators of the financial institution under study. Similarly, the instrument that has served to analyze job performance has obtained a confidence level of 0.82 , which is significant for the research.

The verification of the hypothesis has been verified and demonstrated through the Statistical Model used, it has been concluded that work stress is significantly related to the work performance of the Service promoters of the Magdalena District Agencies, in a Financial Institution, determining a Pearson's Chi square of 0.576. The existence of stress problems in the workers was verified, identifying 4 cases of stress; It can be stated that this has a negative effect on the promoters' job performance, concluding that the consequences affect productivity in each of the agencies where the promoters work.
\end{abstract}

Keywords: work stress; performance; burnout; emotional exhaustion; physiological effect

Artículo recibido: 30 noviembre. 2021

Aceptado para publicación: 29 diciembre 2021

Correspondencia: elizabethdelcarmenvelasquezfue@gmail.com

Conflictos de Interés: Ninguna que declarar 


\section{INTRODUCCIÓN}

Hablar del estrés laboral en la actualidad, es tratar un tema de importancia y trascendencia que poco a poco ha ido tomando mayor presencia en todos los campos laborales. El estrés como estudio clínico presenta una serie de factores desencadenantes en el rendimiento y estabilidad de la persona; en lo laboral, el estrés repercute de manera negativa en la productividad del trabajador. Diversas investigaciones que se citan en el desarrollo de este informe conlleva a determinar que la presencia del estrés en los trabajadores se constituye en un síntoma relacionado con el bajo rendimiento laboral. Por otro lado, se hace indispensable realizar el estudio ya que la consecuencia final del estrés estaría relacionada con el deterioro de la salud del trabajador y a la vez con el desempeño laboral de los trabajadores que son materia de estudio.

El estrés en el trabajo se constituye en una enfermedad silenciosa, donde el trabajador no se da cuenta al momento que la adquiere, empieza a sentir síntomas de cansancio, aburrimiento y rendimiento afectando el desempeño y las labores al trabajador no le salen de la manera correcta; todos estos estresores son los que podrían tener un desencadenamiento negativo y que influiría directamente en la salud del trabajador. En esta investigación, se relaciona la variable estrés laboral con el desempeño, para ello se ha tomado como unidad de investigación una institución financiera de Lima; la inquietud de realizar esta investigación radica desde el punto de vista de la observación, donde se aprecia al personal cansado, las metas no se cumplen y se siente un clima poco dinámico en el personal. Vistas las causas de sospecha ante un problema de bajo rendimiento laboral, es que se hace necesario realizar esta investigación que permitirá medir la relación existente entre una variable y la otra.

Los objetivos de este estudio fueron determinar cómo se relaciona el estrés laboral con el desempeño de los promotores de servicio de las Agencias del distrito de Magdalena, en una Institución Financiera, explicando cómo se relaciona el agotamiento emocional con el desempeño de los promotores, explicando también cómo se relacionan los efectos fisiológicos del estrés con el desempeño de los promotores y finalmente se explica y se comprueba la relación de los logros profesionales con el desempeño de los promotores.

Para el desarrollo del estudio se desarrollaron dos encuestas, una orientada a los promotores de servicio de la Institución Financiera a quienes se les aplicó la prueba del modelo de evaluación de la técnica $270^{\circ}$ a una muestra representativa de 25 promotores, 
de igual forma se aplicó el mismo instrumento a los jefes de las agencias. La prueba psicológica para medir el estrés de aplicó a 25 jefes de la agencia en estudio. La validación de los instrumentos empleados se ha realizado mediante el hallazgo del Alfa de Cronbach, determinándose un nivel de confianza aceptable de los ítems planteados en el instrumento. La importancia de la investigación radica en que se ha conocido el nivel de relación existente entre el estrés y el desempeño laboral de los jefes de las agencias bancarias en estudio. También es importante porque los usuarios de la institución financiera podrán contar con un estudio que les permita tener una idea de la existencia de factores colaterales, como es el estrés, que afecta de alguna manera la productividad de sus trabajadores, y a partir de ello se cuente con un antecedente que plasmar indicadores medibles de manera frecuente y reducir el problema.

\section{Formulación del problema}

En la actualidad las empresas buscan seleccionar su personal bajo el modelo de gestión por competencias, es decir, que estos desarrollen competencias que exige el mercado laboral, aportando mayor conocimiento para la realización de sus tareas y lograr el objetivo propuesto, más no hay un mayor compromiso con el desarrollo global del personal, es decir la empresa realiza seguimientos continuos de lo que logra el empleado basado en la meta a desarrollar, mas no de la situación que tiene que vivir el colaborador para poder alcanzarlo, es decir no existe un mejor acercamiento con el personal, de manera que si la empresa detectaría a tiempo la reacción que puede tener el empleado, podría aportar de mucha ayuda para que no se vea afectado por el estrés laboral y así el empleado pueda desempeñarse sin dificultades al entorno que se enfrenta.

Como introducción al problema, según la encuesta sobre salud laboral aplicada por la empresa GRM (2020) a 200 trabajadores que ocupan altos cargos, aún existe un 17\% de ejecutivos que no reconoce los síntomas que produce el estrés, sin embargo, el 32\% respondió que no sabe cómo combatir este mal, de manera que hace que la situación se ponga más difícil y uno termina conviviendo con el problema hasta que se genera un mal más grave para quien lo enfrenta.

En la investigación de mercado, los ejecutivos que si saben cuándo están pasando por un cuadro de estrés asociado al clima laboral, indican que esto parte del ambiente laboral, en el trabajo bajo presión y en la mala relación con el jefe directo, quiere decir que si no hay una equidad en los colaboradores, no existe una buena relación con el jefe y hay una 
exigencia laboral primordial, simple y llanamente esto lograra que el trabajo que se realice no sea el esperado por la empresa.

La Organización Mundial de la Salud (OMS) (2014) definió al estrés laboral como la reacción que puede tener el individuo ante exigencias y presiones laborales que no se ajustan a sus conocimientos y capacidades y que ponen a prueba su capacidad para afrontar la situación.

Combatir ese mal, puntualiza la OMS, contribuye a un mejor funcionamiento de las empresas, pues un trabajador estresado suele ser más enfermizo, estar poco motivado, ser menos productivo y tener menos seguridad laboral, para lo cual eso implica para la empresa peores perspectivas de éxito en un mercado competitivo, ya que el punto clave del éxito en las organizaciones son las personas, las empresas hacen poco por enfrentarlo con efectividad, es decir algunas si las enfrentan pero no al análisis del fondo, simplemente desarrollan talleres de relajación, mas no recabar más en la información que se requiere para tratar el problema.

Según el estudio, el 92\% de los encuestados asegura que sus empresas no tienen programas para prevenir o disminuir el estrés, y el $70 \%$ que dicen que sí, considera que no son efectivos. En ambos casos se observa que las empresas no priorizan la salud emocional de los empleados, quiere decir que las empresas aun no implementan programas que forme parte del crecimiento personal para los empleados.

Otros estudios, como el realizado por Guic, Bilbao y Col (2012) señalaron que el estrés laboral no sólo libera dificultades en el contorno de la salud mental y física de los trabajadores, sino que también tiene consecuencias económicas para las empresas donde éstos trabajan. Citando el caso anterior no se encuentran estudios para evaluar el costo del estrés ocupacional, pero trabajos realizados en otros países, como Estados Unidos de América e Inglaterra, demuestran que el estrés ocupacional tiene un alto costo tanto individual como organizacional. Otro estudio realizado por Rodríguez (2011) señaló que el campo laboral actual delimitado por la globalidad y la inestabilidad produce ciertas condiciones que hace que buena cantidad de profesionales experimenten síntomas de frustración y un nivel elevado de tensión emocional, lo que conlleva a un aumento de los niveles de estrés laboral y del desgaste profesional que afecta directamente el desempeño laboral. El informe presentado por Rodríguez, demostró que la importancia del estudio del estrés laboral y el burnout tiene una tendencia a incrementar en estos últimos años. 
Otra precisión que se ha podido establecer en la problemática del estrés laboral, es que cuando el estrés llega a su etapa máxima se le denomina "quemado", estudios como el realizado por Quiceno y Vinaccia (2007) realizaron su investigación cuyo objetivo fue revisar la literatura y presentar una aproximación sobre la conceptualización del Burnout o Síndrome de Quemarse en el Trabajo (SQT) haciendo una remembranza en primera instancia a las bases teóricas del estrés y las estrategias de afrontamiento hasta llegar a definir el estrés laboral, el cual da curso al surgimiento del Burnout como "proceso". Por consiguiente, los investigadores hacen referencia de las dimensiones que lo componen, como de la sintomatología física, emocional y conductual que se manifiesta en quienes tiene este síndrome y las características organizacionales que pueden propiciarlo. Como se puede apreciar con esta breve referencia de estudios similares, es notable que la investigación realizada tiene una connotación no solo en el ámbito local sino también internacional, en tal sentido el problema es de connotación universal y que se puede suscitar en diferentes empresas como las financieras.

Por otro lado, se debe tomar en cuenta que el reto actual de la dirección y gestión de los Recursos Humanos radica en crear herramientas útiles mediante las cuales el capital humano pueda llegar a comprometerse con los objetivos organizacionales e integrarse en el proyecto de empresa, para obtener ventajas sostenibles y duraderas en el tiempo. De esta manera, altos niveles de compromiso posibilitarán que se mantengan las capacidades colectivas generadas y las ventajas sostenibles y duraderas que la organización ha sido capaz de lograr y por ende reflejarlo en el desempeño.

Sin embargo; en los últimos años se ha podido observar que las instituciones tanto privadas como públicas de nuestro país se han visto afectadas por un fenómeno que está alterando el sistema laboral, esto comienza a preocupar a los empleadores, y a los propios organismos del estado, ya que se ha observado una crisis en la adaptación a las exigencias de la dedicación y vocación del personal, entre los problemas que estos aquejan se observa que muchos de ellos han convertido su desempeño en una rutina laboral, dejando de lado la importancia que amerita la calidad del trabajo que realizan.

Este problema que en las últimas décadas ha tomado la denominación de estrés laboral, el cual evidencia una serie de respuestas emocionales y conductuales entre ellas tenemos: pobre control de impulsos, irritabilidad, baja tolerancia a la frustración, hipersensibilidad a la crítica, apatía, olvidos frecuentes, tardanzas, absentismo laboral, relaciones 
interpersonales deficientes; si bien es cierto esta sintomatología arrastra en sí misma un déficit en la productividad tanto cualitativa como cuantitativa, así mismo afecta al logro de objetivos grupales y a la identificación con la organización viéndose afectado hasta la productividad y objetivos de la organización.

Por otro lado, en relación a la productividad que vienen demostrando los empleados de la institución financiera en estudio, se tiene de conocimiento que en el año 2019 el cumplimiento de las metas se redujo en $12 \%$ en relación al 2020. Este es un problema que radica puntualmente en el desempeño laboral de los empleados, es preocupante para los encargados de la dirección de la institución financiera conocer las causas del problema. Sin embargo, se evidencia como principal causa, el bajo nivel de productividad de los empleados de la institución financiera debido a factores relacionados con la salud.

El lugar investigado ha sido la agencia de Magdalena de una Institución Financiera de Lima, debido a que el distrito es una zona comercial, y la oficina se encuentra alrededor de un gran flujo de comercio, teniendo una gran cantidad de afluencia de público, cabe resaltar que esta oficina muchas veces no se da abasto debido que es una oficina pequeña donde los clientes sobrepasan el aforo y donde el colaborador se siente presionado por las diferentes situaciones que se presenten.

Literalmente, para la empresa supone un mayor índice de absentismo, rotaciones excesivas, incluso abandono del puesto por parte del trabajador, traduciéndose en deterioro del rendimiento y de la calidad (Maslach y Leiter, 2009, pág. 32). La investigación fue realizada en el periodo 2019 y Julio del 2020, recolectando datos de los promotores de servicio que tienen entre 1 a 3 años en el puesto para analizar la influencia que tienen los niveles de estrés laboral en los resultados. Así, ante lo expuesto, la investigación responderá a las siguientes preguntas:

\section{Problema principal:}

¿Cómo se relaciona el estrés laboral con el desempeño laboral de los promotores de servicio de las agencias de una institución financiera del distrito de Magdalena?

\section{ESTRATEGIAS METODOLÓGICAS O MATERIALES Y MÉTODOS}

La presente investigación utilizó un diseño del tipo descriptivo - correlacional ya que tuvo como finalidad conocer y determinar las diferentes situaciones de estrés a las que están expuestas los trabajadores de una Institución Financiera de Lima, sobretodo cajeros, entre estas situaciones tenemos: el manejo de dinero, la exposición a posibles estafas, las 
metas de ventas que tienen y el manejo de clientes complicados; entre otros problemas diarios que surgen durante la atención que se brinda a los clientes en esta entidad.

Asimismo, el estudio es de tipo cuantitativo porque se analizó la información obtenida con la aplicación de los instrumentos; y cualitativo en la medida que estos resultados se compararon con las teorías de los diversos autores y permitió llegar a conclusiones o resultados de la investigación. Se aplicó este diseño porque se recopiló directamente la información de los trabajadores de la entidad bancaria, lo que permitió obtener información directa y de primera mano a fin de determinar las deficiencias, mejoras y optimización de las condiciones de trabajo y desempeño laboral de los trabajadores de la institución financiera. En estos diseños lo que se mide es la relación entre variables en un tiempo determinado.

Por lo tanto, los diseños correlacionales/causales pueden limitarse a establecer relaciones entre variables sin precisar sentido de causalidad o pueden pretender analizar relaciones de causalidad. Cuando se limitan a relaciones no causales, se fundamentan en hipótesis correlacionales y cuando buscan evaluar relaciones causales, se basan en hipótesis causales.

\section{Población y muestra}

En esta investigación se determinó como población al personal operativo que trabaja en las agencias de la institución financiera ubicadas en el distrito de Magdalena del Mar, distribuido de la siguiente manera.

\begin{tabular}{|l|c|}
\hline \multicolumn{1}{|c|}{ Agencia } & $\begin{array}{c}\text { Cantidad de Personal Operario atención } \\
\text { al cliente }\end{array}$ \\
\hline Calle José Valencia 575 & 12 \\
\hline Av. Alberto del Campo 1010 & 14 \\
\hline Av. J. Prado Oeste 575 & 6 \\
\hline Av. Salaverry Esq. Av. Del Ejército & 16 \\
\hline \multicolumn{1}{|c|}{ TOTAL } & $\mathbf{4 8}$ personas \\
\hline
\end{tabular}

En total hay 48 personas que atienden al cliente en las ventanillas de atención al cliente en el distrito de Magdalena del Mar en la institución financiera de Lima. También se consideró necesario que los instrumentos se apliquen a los funcionarios, que tienen otra visión o perspectiva del problema, entre ellos tenemos:

- Gerentes (3)

- Sub gerentes (2)

- Jefes de agencia (4). 
- Supervisores (8).

- Personal de plataforma (18)

El tamaño de la población ascendió a 83 personas según la distribución antes indicada. A pesar de que se debe utilizar el muestreo probabilístico y los criterios de inclusión y exclusión para la investigación de acuerdo a las exigencias metodológicas, se procedió a indicar los criterios, por lo tanto se ha tomado de manera aleatoria 25 promotores y 25 jefes de las agencias:

- Sexo: masculino y femenino

- Edades comprendidas entre 18 y 60 años de edad.

- Nivel de formación: técnico y universitario (pregrado, maestría y doctorado).

\section{Técnicas e instrumentos}

En la presente investigación se emplearon dos instrumentos, uno orientado a los promotores de las agencias y el segundo a los jefes. En primer lugar, se aplicó la encuesta dirigida al personal operativo (atención en ventanilla al cliente) y también se aplicó la encuesta dirigida a los funcionarios y personal directivo de la Institución Bancaria que trabajan en las agencias del distrito de Magdalena. Dicho instrumento (Encuesta fue elaborado en base a los objetivos de la investigación y sus respectivos indicadores y dimensiones de manera que nos permitió recoger información sobre el tema de investigación).

\section{RESULTADOS Y DISCUSIÓN}

El tema de la relación del estrés con el desempeño laboral de los trabajadores de una institución financiera, es motivo de gran preocupación por las repercusiones en el ámbito personal y de la organización en sus estudios como: ausentismo, disminución del nivel de rendimiento, alta movilidad laboral, repercusión familiar, etc. Desde el punto de vista observacional, se han identificado numerosos factores estresantes crónicos del entorno laboral implicados en su producción profesional, como: la sobrecarga de tareas, incentivos, calidad de las relaciones en equipo.

Tabla 1. Resultados de la variable agotamiento emocional

\begin{tabular}{cccc}
\hline & F & \% & Hi \\
\hline Bajo & 5 & 21 & 21 \\
Medio & 14 & 56 & 77 \\
Alto & 6 & 23 & 100 \\
\hline Total & $\mathbf{2 5}$ & $\mathbf{1 0 0 . 0 0}$ & \\
\hline
\end{tabular}

Fuente: Resultados de la encuesta - Febrero 2020 
De acuerdo a la Tabla 1 se aprecia que el $21 \%$ de los promotores presentaron un nivel de agotamiento bajo, el 56\% muestran un nivel de agotamiento medio y el $23 \%$ de los promotores tienen agotamiento alto (representado por 6 promotores), lo cual es un indicador alto que debe ser tratado de manera urgente.

Por otro lado, en la Tabla 2, se apreció la relación de los resultados entre las dos variables.

Tabla 2 Relación entre la variable agotamiento emocional y el desempeño de los promotores de la IF

\begin{tabular}{lcccccccccc}
\hline $\begin{array}{l}\text { Agotamiento } \\
\text { Emocional }\end{array}$ & $\mathbf{F}$ & $\mathbf{\%}$ & Bajo & $\mathbf{\%}$ & Medio & $\mathbf{\%}$ & Alto & $\mathbf{\%}$ & TOTAL & $\boldsymbol{\%}$ \\
\hline Bajo & 5 & 21 & 2 & 7 & - & - & - & - & 6 & 12 \\
Medio & 14 & 56 & - & - & 8 & 30 & - & - & 22 & 44 \\
Alto & 6 & 23 & - & - & - & - & 16 & 63 & 22 & 44 \\
\hline Total & $\mathbf{2 5}$ & $\mathbf{1 0 0}$ & $\mathbf{2}$ & $\mathbf{7}$ & $\mathbf{8}$ & $\mathbf{3 0}$ & $\mathbf{1 6}$ & $\mathbf{6 3}$ & $\mathbf{5 0}$ & $\mathbf{1 0 0}$ \\
\hline Chi - cuadrado de Pearson $=0.661 g . l .=$ & 12 & $* * *<.001$ & & & \\
Correlación de Spearman $=0.701$
\end{tabular}

Fuente: Resultados de la encuesta - Febrero 2020

Como se muestra en la tabla 2, el agotamiento emocional está relacionado directamente con el Desempeño Laboral, según la correlación de Spearman de 0.701, representando ésta una aceptable asociación de las variables y siendo altamente significativo. Además, según la prueba de la independencia (Chi-cuadrado: ***p <.001) altamente significativo, se acepta la relación de las variables establecidas. Consecuentemente, la correlación de Pearson de 0.66 demuestra que existe una relación directamente significativa en las variables analizadas. Porcentualmente se puede determinar que el $44 \%$ de los promotores se encuentran en un nivel alto de agotamiento emocional frente al desempeño laboral, otro $44 \%$ presentan un nivel medio y el $12 \%$ de promotores están en un nivel bajo.

\section{Los efectos fisiológicos del estrés con el desempeño de los promotores.}

En el ámbito teórico y de investigaciones acerca del tema del estrés, existe una amplia información. Distintos investigadores indican que el estrés es una respuesta inesperada del organismo ante un estímulo inespecífico; tratándose de una tensión a casusa de situaciones agobiantes, las cuales tienen reacciones psicosomáticas o trastornos psicológicos (Sandín, 1995; Keith, 1994).

En la Tabla 3, se puede apreciar que el 58\% de los promotores presentan efectos fisiológicos del estrés en un nivel bajo, el $24 \%$ de los promotores lo relacionan con un nivel medio y el $18 \%$ se relaciona con un nivel alto. 
Tabla 3 Resultados de la variable efectos fisiológicos

\begin{tabular}{cccc}
\hline & F & \% & Hi \\
\hline Bajo & 14 & 58 & 58 \\
Medio & 6 & 24 & 82 \\
Alto & 5 & 18 & 100 \\
\hline Total & $\mathbf{2 5}$ & $\mathbf{1 0 0 . 0 0}$ & \\
\hline
\end{tabular}

Fuente: Resultados de la encuesta - Febrero 2020

A continuación, en la Tabla 4, se presenta la relación entre la variable efectos fisiológicos y el desempeño de los promotores de la Institución Financiera, donde se describe el análisis de la relación de manera descriptiva e inferencial.

Tabla 4 Relación entre la variable efectos fisiológicos y el desempeño de los promotores de la IF

\begin{tabular}{ccccccccccc}
\hline \multirow{2}{*}{$\begin{array}{c}\text { Efectos } \\
\text { Fisiológicos }\end{array}$} & $\mathbf{F}$ & $\mathbf{\%}$ & Bajo & $\mathbf{\%}$ & Medio & $\mathbf{\%}$ & Alto & $\mathbf{\%}$ & TOTAL & \% \\
\hline Bajo & 14 & 58 & 2 & 7 & - & - & - & - & 16 & 32 \\
Medio & 6 & 24 & & & 8 & 30 & - & - & 13 & 27 \\
Alto & 5 & 18 & & & - & - & 16 & 63 & 20 & 41 \\
\hline Total & $\mathbf{2 5}$ & $\mathbf{1 0 0}$ & $\mathbf{2}$ & $\mathbf{7}$ & $\mathbf{8}$ & $\mathbf{3 0}$ & $\mathbf{1 6}$ & $\mathbf{6 3}$ & $\mathbf{5 0}$ & $\mathbf{1 0 0}$ \\
\hline Chi - cuadrado de Pearson $=0.621$ lg.l. & $=$ & 12 & $* * *<.001$ & & & \\
Correlación de Spearman $=0.648$ & & & & & & \\
\hline
\end{tabular}

Fuente: Resultados de la encuesta - Febrero 2020

Como se muestra en la tabla 4, los efectos fisiológicos del estrés están relacionados directamente con el Desempeño Laboral, según la correlación de Spearman de 0.648, representando ésta una aceptable asociación de las variables y siendo altamente significativo. Además, según la prueba de la independencia (Chi-cuadrado: $* * * p<.001$ ) altamente significativo, se acepta la relación de las variables establecidas. Consecuentemente, la correlación de Pearson de 0.62 demuestra que existe una relación directamente significativa en las variables analizadas. Porcentualmente se puede determinar que el $41 \%$ de los promotores se encuentran en un nivel alto sobre los efectos fisiológicos frente al desempeño laboral, otro $27 \%$ presentan un nivel medio y el $32 \%$ de promotores están en un nivel bajo.

\section{Los logros profesionales y el desempeño de los promotores de Servicio}

En la Tabla 5, de manera frecuencial, el 8\% de los promotores presentan un nivel bajo de los logros profesionales. El 38\% presentan un nivel medio y el 54\% un nivel alto. 
Tabla 5 Resultados de la variable Logros Profesionales

\begin{tabular}{cccc}
\hline & F & \% & Hi \\
\hline Bajo & 2 & 8 & 8 \\
Medio & 10 & 38 & 46 \\
Alto & 14 & 54 & 100 \\
\hline Total & $\mathbf{2 5}$ & $\mathbf{1 0 0}$ & \\
\hline
\end{tabular}

Fuente: Resultados de la encuesta - Febrero 2020

A continuación, en la Tabla 6, se presenta la relación de las variables logros profesionales y el desempeño de los promotores de la IF.

Tabla 6 Relación entre la variable logros profesionales y el desempeño de los promotores de la IF

\begin{tabular}{lcccccccccc}
\hline \multicolumn{1}{c}{$\begin{array}{c}\text { Logros } \\
\text { Profesionales }\end{array}$} & $\mathbf{F}$ & $\mathbf{\%}$ & Bajo & $\mathbf{\%}$ & Medio & $\mathbf{\%}$ & Alto & $\mathbf{\%}$ & TOTAL & \% \\
\hline Bajo & 2 & 8 & 2 & 7 & - & - & - & - & 3 & 6 \\
Medio & 10 & 38 & - & - & 8 & 30 & - & - & 17 & 35 \\
Alto & 14 & 54 & - & - & - & - & 16 & 63 & 30 & 59 \\
\hline \multicolumn{1}{r}{ Total } & $\mathbf{2 5}$ & $\mathbf{1 0 0}$ & $\mathbf{2}$ & $\mathbf{7}$ & $\mathbf{8}$ & $\mathbf{3 0}$ & $\mathbf{1 6}$ & $\mathbf{6 3}$ & $\mathbf{5 0}$ & $\mathbf{1 0 0}$ \\
\hline Chi - cuadrado de Pearson $=0.622$ & g.l. $=$ & 6 & $* * *<.001$ & & & \\
Correlación de Spearman $=0.612$ & & & & & & \\
\hline
\end{tabular}

Fuente: Resultados de la encuesta - Febrero 2020

Como se muestra en la tabla 6, el logro profesional está relacionado directamente con el Desempeño Laboral, según la correlación de Spearman de 0.612, representando ésta una aceptable asociación de las variables y siendo altamente significativo. Además, según la prueba de la independencia (Chi-cuadrado: $* * * p<.001$ ) altamente significativo, se acepta la relación de las variables establecidas. Consecuentemente, la correlación de Pearson de 0.612 demuestra que existe una relación directamente significativa en las variables analizadas. Porcentualmente se puede determinar que el 59\% de los promotores se encuentran en un nivel alto de logros profesionales frente al desempeño laboral, otro $35 \%$ presentan un nivel medio y el $6 \%$ de promotores están en un nivel bajo.

\section{Relación entre el estrés laboral con el desempeño de los promotores}

Para demostrar el objetivo general planteado, se presentan los resultados obtenidos de manera independiente, en la Tabla 7 se puede apreciar que $23 \%$ de los promotores tiene un nivel de estrés bajo, el $57 \%$ presentan estrés laboral medio y el $20 \%$ tienen estrés laboral alto. 
Tabla 7 Resultados de la variable Estrés Laboral

\begin{tabular}{cccc}
\hline & F & \% & Hi \\
\hline Bajo & 6 & 23 & 23 \\
Medio & 14 & 57 & 80 \\
Alto & 5 & 20 & 100 \\
\hline Total & $\mathbf{2 5}$ & $\mathbf{1 0 0 . 0 0}$ & \\
\hline
\end{tabular}

Fuente: Resultados de la encuesta - Febrero 2020

A continuación, en la Tabla 8, se presenta la descripción de los resultados de la variable Desempeño Laboral.

Tabla 8 Resultados de la variable Desempeño Laboral

\begin{tabular}{cccc}
\hline & F & \% & Hi \\
\hline Bajo & 2 & 7 & 7 \\
Medio & 8 & 30 & 37 \\
Alto & 16 & 63 & 100 \\
\hline Total & $\mathbf{2 5}$ & $\mathbf{1 0 0}$ & \\
\hline
\end{tabular}

Fuente: Resultados de la encuesta - Febrero 2020

A continuación, en la Tabla 9, se presentan los resultados que pueden comprobar la hipótesis general planteada, determinándose que existe una relación directa - significativa entre la entre la variable estrés laboral y el desempeño de los promotores de la IF.

Tabla 9 Relación entre la variable estrés laboral y el desempeño de los promotores de la $I F$

\begin{tabular}{ccccccccccc}
\hline Estrés & \multicolumn{10}{c}{ Desempeño Laboral } \\
\cline { 2 - 11 } Laboral & $\mathbf{F}$ & $\mathbf{\%}$ & Bajo & $\mathbf{\%}$ & Medio & $\mathbf{\%}$ & Alto & $\mathbf{\%}$ & TOTAL & \% \\
\hline Bajo & 6 & 23 & 2 & 7 & & & & & 8 & 15 \\
Medio & 14 & 57 & & & 8 & 30 & & & 22 & 43 \\
Alto & 5 & 20 & & & & & 16 & 63 & 21 & 42 \\
\hline Total & $\mathbf{2 5}$ & $\mathbf{1 0 0}$ & $\mathbf{2}$ & $\mathbf{7}$ & $\mathbf{8}$ & $\mathbf{3 0}$ & $\mathbf{1 6}$ & $\mathbf{6 3}$ & $\mathbf{5 0}$ & $\mathbf{1 0 0}$ \\
\hline Chi - cuadrado de Pearson $=0.576$ & g.l. $=$ & 16 & $* * *<.001$ & & & \\
Correlación de Spearman $=0.595$ & & & & & & & \\
\hline
\end{tabular}

Fuente: Resultados de la encuesta - Febrero 2020

Como se muestra en la Tabla 9, el Estrés Laboral está relacionado directamente con el Desempeño Laboral, según la correlación de Spearman de 0.595, representando ésta una aceptable asociación de las variables y siendo altamente significativo. Además, según la prueba de la independencia (Chi-cuadrado: $* * * p<.001$ ) altamente significativo, se acepta la relación de las variables establecidas. Consecuentemente, la correlación de Pearson de 0.576 demuestra que existe una relación directa significativa en las variables 
analizadas. Porcentualmente se puede determinar que el $42 \%$ de los promotores se encuentran en un nivel alto de estrés laboral frente al desempeño laboral, otro $43 \%$ presentan un nivel medio y el $15 \%$ de promotores están en un nivel bajo.

\section{Resultados descriptivos del desempeño de los Jefes}

En la Tabla 10, se aprecian los resultados relacionados con la adaptación a nuevas situaciones y cambios que producen sus actividades diarias con una actitud positiva frente a ello. El 24\% indican que casi nunca, el $36 \%$ señalan que pocas veces, el $20 \%$ indican que algunas veces, el 16\% indican que casi siempre y el $4 \%$ señalan que siempre.

\section{Tabla 10}

Se adapta a nuevas situaciones y cambios que se producen sus actividades diarias con una actitud positiva frente a ello.

\begin{tabular}{|ll|r|r|r|r|}
\hline & & & & \multicolumn{2}{|c|}{$\begin{array}{c}\text { Porcentaje } \\
\text { acumulado }\end{array}$} \\
\hline Válidos & Casi Nunca & 6 & 24,0 & 24,0 & 24,0 \\
& Pocas veces & 9 & 36,0 & 36,0 & 60,0 \\
& Algunas & 5 & 20,0 & 20,0 & 80,0 \\
veces & & & & 96,0 \\
Casi siempre & 4 & 16,0 & 16,0 & 100,0 \\
Siempre & 1 & 4,0 & 4,0 & \\
Total & 25 & 100,0 & 100,0 & Porcentaje \\
\hline
\end{tabular}

Fuente: Resultados de la encuesta - Febrero 2020

En la Tabla 11, se aprecian los resultados relacionados con la aceptación de nuevas formas de realizar su trabajo y escucha otros puntos de vista para mejorar su desempeño diario. El $4 \%$ indican que casi nunca, el $28 \%$ señalan que pocas veces, el $20 \%$ indican que algunas veces, el $40 \%$ indican que casi siempre y el $8 \%$ señalan que siempre.

\section{Tabla 11}

Acepta nuevas formas de realizar su trabajo y escucha otros puntos de vista para mejorar su desempeño diario.

\begin{tabular}{|ll|r|r|r|r|}
\hline & & & & Porcentaje \\
& & Frecuencia & Porcentaje & Porcentaje válido & acumulado \\
\hline Válidos & 1 & 4,0 & 4,0 & 4,0 \\
& Cunca & 28,0 & 28,0 & 32,0 \\
& 7 & 20,0 & 20,0 & 52,0 \\
Pocas veces & 5 & 40,0 & 40,0 & 92,0 \\
Algunas veces & 10 & 8,0 & 8,0 & 100,0 \\
Casi siempre & 2 & 100,0 & 100,0 & \\
Total & 25 & &
\end{tabular}

Fuente: Resultados de la encuesta - Febrero 2020 
En la Tabla 12, se aprecian los resultados relacionados con la preocupación por realizar mejoras en la ejecución de sus actividades y aumentar sus niveles de productividad. El $4 \%$ indican que casi nunca, el $20 \%$ señalan que pocas veces, el $36 \%$ indican que algunas veces, el $12 \%$ indican que casi siempre y el $4 \%$ señalan que siempre.

\section{Tabla 12}

Se preocupa realizar mejoras en la ejecución de sus actividades y aumentar sus niveles de

\begin{tabular}{|ll|r|r|r|r|}
\hline \multicolumn{1}{|c|}{ productividad. } \\
& & & & \multicolumn{2}{c|}{$\begin{array}{c}\text { Porcentaje } \\
\text { acumulado }\end{array}$} \\
\hline Válidos & Frecuencia & Porcentaje & Porcentaje válido & 4,0 \\
& Nunca & 1 & 4,0 & 4,0 & 24,0 \\
& Casi Nunca & 5 & 20,0 & 20,0 & 60,0 \\
Pocas veces & 9 & 36,0 & 36,0 & 84,0 \\
Algunas veces & 6 & 24,0 & 24,0 & 96,0 \\
Casi siempre & 3 & 12,0 & 12,0 & 100,0 \\
Siempre & 1 & 4,0 & 4,0 & \\
Total & 25 & 100,0 & 100,0 & \\
\hline
\end{tabular}

Fuente: Resultados de la encuesta - Febrero 2020

En la Tabla 13, se aprecian los resultados relacionados con la solución a dificultades que se presentan en el día para terminar con éxito su trabajo. El 12\% indican que casi nunca, el $16 \%$ señalan que pocas veces, el $32 \%$ indican que algunas veces, el $28 \%$ indican que casi siempre y el $12 \%$ señalan que siempre.

\section{Tabla 13}

Soluciona dificultades que se presentan en el día a día para terminar con éxito su trabajo.

\begin{tabular}{|l|r|r|r|r|}
\hline & Frecuencia & Porcentaje & Porcentaje válido & Porcentaje acumulado \\
\hline Válidos Nunca & 3 & 12,0 & 12,0 & 12,0 \\
Casi Nunca & 4 & 16,0 & 16,0 & 28,0 \\
Pocas veces & 8 & 32,0 & 32,0 & 60,0 \\
Algunas veces & 7 & 28,0 & 28,0 & 88,0 \\
Casi siempre & 3 & 12,0 & 12,0 & 100,0 \\
Total & 25 & 100,0 & 100,0 & \\
\hline
\end{tabular}

Fuente: Resultados de la encuesta - Febrero 2020

En la Tabla 14, se aprecian los resultados relacionados con la concentración de tareas que le demandan mayor esfuerzo para cumplir a tiempo con lo solicitado. El 12\% indican que casi nunca, el $20 \%$ señalan que pocas veces, el $24 \%$ indican que algunas veces, el $32 \%$ indican que casi siempre y el $12 \%$ señalan que siempre. 


\section{Tabla 14}

Se concentra en las tareas que le demandan mayor esfuerzo para cumplir a tiempo con lo solicitado.

\begin{tabular}{|ll|r|r|r|r|}
\hline & Frecuencia & Porcentaje & Porcentaje válido & Porcentaje acumulado \\
\hline Válidos & Nunca & 3 & 12,0 & 12,0 & 12,0 \\
& Casi Nunca & 5 & 20,0 & 20,0 & 32,0 \\
Pocas veces & 6 & 24,0 & 24,0 & 56,0 \\
Algunas veces & 8 & 32,0 & 32,0 & 88,0 \\
Casi siempre & 3 & 12,0 & 12,0 & 100,0 \\
Total & 25 & 100,0 & 100,0 & \\
\hline
\end{tabular}

Fuente: Resultados de la encuesta - Febrero 2020

En la Tabla 15, se aprecian los resultados relacionados con la percepción e identificación del compromiso hacia la empresa procurando el bienestar de la organización. El 24\% indican que casi nunca, el $32 \%$ señalan que pocas veces, el $28 \%$ indican que algunas veces, el $12 \%$ indican que casi siempre y el $4 \%$ señalan que siempre.

\section{Tabla 15}

Se puede percibir su identificación y compromiso hacia la empresa, procura el bienestar

\begin{tabular}{|ll|r|r|r|r|}
\hline & & & & \multicolumn{2}{c|}{$\begin{array}{c}\text { Porcentaje } \\
\text { acumulado }\end{array}$} \\
\hline Válidos & Frecuencia & Porcentaje & Porcentaje válido & 24,0 \\
& Casi Nunca & 6 & 24,0 & 24,0 & 56,0 \\
& Pocas veces & 8 & 32,0 & 32,0 & 84,0 \\
Algunas veces & 7 & 28,0 & 28,0 & 96,0 \\
Casi siempre & 3 & 12,0 & 12,0 & 100,0 \\
Siempre & 1 & 4,0 & 4,0 & \\
Total & 25 & 100,0 & 100,0 & \\
\hline
\end{tabular}

Fuente: Resultados de la encuesta - Febrero 2020

En la Tabla 16, se aprecian los resultados relacionados con la actuación con calma y mantiene el ritmo constante aun ante jornadas prolongadas de trabajo sin afectar el rendimiento esperado. El $8 \%$ indican que casi nunca, el 16\% señalan que pocas veces, el $36 \%$ indican que algunas veces, el $24 \%$ indican que casi siempre y el $16 \%$ señalan que siempre. 


\section{Tabla 16}

Actúa con calma y mantiene un ritmo constante aún ante jornadas prolongadas de trabajo sin afectar el rendimiento esperado.

\begin{tabular}{|ll|r|r|r|r|}
\hline & & & \multicolumn{2}{c|}{$\begin{array}{c}\text { Porcentaje } \\
\text { válido }\end{array}$} & \multicolumn{2}{c|}{$\begin{array}{c}\text { Porcentaje } \\
\text { acumulado }\end{array}$} \\
\hline Válidos & Frecuencia & Porcentaje & 8,0 & 8,0 \\
& Canca & 2 & 8,0 & 16,0 & 24,0 \\
& 4 & 16,0 & 36,0 & 60,0 \\
Pocas veces & 9 & 36,0 & 24,0 & 84,0 \\
Algunas & 6 & 24,0 & & \\
veces & & & 16,0 & 100,0 \\
Casi siempre & 4 & 16,0 & 100,0 & \\
Total & 25 & 100,0 & & \\
\hline
\end{tabular}

Fuente: Resultados de la encuesta - Febrero 2020

En la Tabla 17, se aprecian los resultados relacionados con la eficiencia en situaciones difíciles o ante condiciones hostiles que pueden presentarse en su día a día. El 28\% indican que casi nunca, el 24\% señalan que pocas veces, el $40 \%$ indican que algunas veces y el $8 \%$ indican que casi siempre.

\section{Tabla 17}

Es eficiente aun en situaciones difíciles o ante condiciones hostiles que puedan presentarse

\begin{tabular}{|ll|r|r|r|r|}
\hline & & & & \multicolumn{2}{c|}{$\begin{array}{c}\text { Porcentaje } \\
\text { acumulado }\end{array}$} \\
\hline Válidos su día a día \\
& Casi Nunca & 7 & 28,0 & 28,0 & 28,0 \\
& Pocas veces & 6 & 24,0 & 24,0 & 52,0 \\
Algunas veces & 10 & 40,0 & 40,0 & 92,0 \\
Casi siempre & 2 & 8,0 & 8,0 & 100,0 \\
Total & 25 & 100,0 & 100,0 & \\
\hline
\end{tabular}

En la Tabla 18, se aprecian los resultados relacionados con la responsabilidad en sus actividades diarias, asumiendo sus éxitos y errores personales. El $40 \%$ indican que casi nunca, el $20 \%$ señalan que pocas veces, el $32 \%$ indican que algunas veces y el $8 \%$ indican que casi siempre. 


\section{Tabla 18}

Es responsable en sus actividades diarias, asumiendo sus éxitos y errores personales.

\begin{tabular}{|c|r|r|r|r|}
\hline & Frecuencia & Porcentaje & Porcentaje válido & Porcentaje acumulado \\
\hline Válidos Casi Nunca & 10 & 40,0 & 40,0 & 40,0 \\
Pocas veces & 5 & 20,0 & 20,0 & 60,0 \\
Algunas veces & 8 & 32,0 & 32,0 & 92,0 \\
Casi siempre & 2 & 8,0 & 8,0 & 100,0 \\
Total & 25 & 100,0 & 100,0 & \\
\hline
\end{tabular}

Fuente: Resultados de la encuesta - Febrero 2020

En la Tabla 19, se aprecian los resultados relacionados con el mantenimiento de los niveles de desempeño aun en situaciones exigentes y con presión de tiempo. El 8\% indican que nunca, el $32 \%$ indican que casi nunca, el $24 \%$ señalan que pocas veces, el $32 \%$ indican que algunas veces y el $4 \%$ indican que casi siempre.

\section{Tabla 19}

Mantiene sus niveles de desempeño aun en situaciones exigentes y con presión de tiempo.

\begin{tabular}{|l|r|r|r|r|}
\hline & Frecuencia & Porcentaje & Porcentaje válido & Porcentaje acumulado \\
\hline Válidos Nunca & 2 & 8,0 & 8,0 & 8,0 \\
Casi Nunca & 8 & 32,0 & 32,0 & 40,0 \\
Pocas veces & 6 & 24,0 & 24,0 & 64,0 \\
Algunas veces & 8 & 32,0 & 32,0 & 96,0 \\
Casi siempre & 1 & 4,0 & 4,0 & 100,0 \\
Total & 25 & 100,0 & 100,0 & \\
\hline
\end{tabular}

Fuente: Resultados de la encuesta - Febrero 2020

\section{DISCUSIÓN}

El estrés laboral es uno de los problemas más graves que afectan al mundo laboral, y con mayor frecuencia en organizaciones ligadas a la salud, educación y financieras que son por lo general organizaciones donde trabajan brindando servicios como eje y giro del negocio; con enormes consecuencias tanto personales como económicas. Puede afectar a la salud física y mental, dando lugar a alteraciones psicosomáticas (cardio-respiratorias, jaquecas, ulceras, insomnio, etc.), estados ansiosos, depresivos, alcoholismo, etc. (Bronsberg y Vestlund, 1997; Buceta y Bueno, 1995). Para la Institución Financiera supone un mayor índice de absentismo, rotaciones excesivas, incluso abandono del puesto 
por parte del trabajador, traduciéndose en deterioro del rendimiento y de la calidad (Maslach y Leiter, 2018).

Lógicamente, lograr una organización sin estrés laboral, puede parecernos una utopía, pero tenemos que trabajar para que esta enfermedad aparezca lo menos posible en nuestra empresa y así no se convierta en una de las enfermedades profesionales del siglo XXI. Definido como una respuesta al estrés laboral crónico, parece afectar en mayor o menor medida a un colectivo importante. La desmotivación, el desinterés, el malestar interno o la insatisfacción laboral, son algunos de los aspectos que caracterizan el síndrome. (Herbert Freudenberger 1974; Christina Maslach 1976).

No hay un consenso global para explicar este síndrome y una de las teorías más aceptadas como consenso internacional es la proporcionada por Maslach (1976), en la que estaría caracterizado en tres dimensiones: el agotamiento emocional, la despersonalización y la realización personal. Lo anterior involucra una serie de conductas como: el deterioro del rendimiento, la pérdida de responsabilidad, actitudes pasivo-agresivas con los pacientes y pérdida de la motivación; en los que se implican factores internos y externos en torno al personal que lo padece.

El tema de la relación del estrés laboral y el desempeño de los trabajadores de la Institución Financiera, es motivo de gran preocupación por las repercusiones en el ámbito personal y de la organización en sus estudios como: ausentismo, disminución del nivel de rendimiento y de las enfermeras, alta movilidad laboral, repercusión familiar, etc. Desde el punto de vista observacional, se han identificado numerosos factores estresantes crónicos del entorno laboral implicados en su producción profesional, como: la sobrecarga de tareas, incentivos, calidad de las relaciones en equipo.

De acuerdo a lo mencionado, el gran reto actual de la dirección y gestión de los Recursos Humanos radica en crear herramientas útiles mediante las cuales el personal pueda llegar a comprometerse con los objetivos organizacionales e integrarse en el proyecto de empresa, para obtener ventajas sostenibles y duraderas en el tiempo. El desempeño laboral puede ser uno de los mecanismos que tiene la Dirección de Recursos Humanos en la Institución Financiera que permite analizar la lealtad y vinculación de los empleados con la organización. Así, si se consigue que los empleados estén muy identificados e implicados en la organización en la que trabajan (compromiso actitudinal), mayores serán las probabilidades de que permanezcan en la misma. Por otra parte, se puede intentar que 
los individuos se deban a la organización en base a sus propios intereses (compromiso de continuidad). De esta manera, altos niveles de compromiso posibilitarán que se mantengan las capacidades colectivas generadas y las ventajas sostenibles y duraderas que la organización ha sido capaz de lograr.

Debido a la falta de mayores estudios en el país, es de gran relevancia investigar sobre estrés laboral tomando como muestra los trabajadores que laboran en la Institución Financiera, ya que puede ofrecer aportes orientados a satisfacer las necesidades de los profesionales, así como también, entender la asociación de ciertos factores. En cuanto al agotamiento emocional en los trabajadores de la Institución Financiera, se alude a la situación en que ellos sienten que ya no pueden dar más de sí mismos a nivel laboral; es una situación de agotamiento de la energía o los recursos emocionales propios, una experiencia de estar emocionalmente agotado debido al contacto "diario" y mantenido con personas a las que hay que atender como objeto de trabajo realizado en las prácticas profesionales.

Por lo expuesto anteriormente y debido a lo preocupante que resulta esta patología en el sistema laboral de la Institución Financiera, se decide realizar este estudio con el propósito de investigar la existencia del síndrome en los promotores que laboran en la Institución Financiera, para así poder definir las variables sociales más características e identificar los síntomas que manifiestan una mayor cercanía a presentar la enfermedad dentro de los profesionales de la organización en estudio, con ello se podrán dar pautas claras de intervención precoz, necesarias para la mejoría de la atención que más nos preocupa: los promotores que tiene la Institución Financiera.

\section{CONCLUSIÓN O CONSIDERACIONES FINALES}

1. Se ha podido determinar que el estrés laboral se relaciona significativamente con el desempeño laboral de los promotores de Servicio de las Agencias del Distrito de Magdalena, en una Institución Financiera. Se ha comprobado la existencia de problemas de estrés en los trabajadores, identificándose 4 casos de estrés; pudiéndose afirmar que ello repercute de manera negativa en el desempeño laboral de los promotores, concluyéndose que las consecuencias afectan la productividad en cada una de las agencias donde laboran los promotores.

2. Se ha comprobado que el agotamiento emocional se relaciona significativamente con el desempeño de los promotores, si se han determinado 4 casos de estrés, ello es 
congruente con los resultados estadísticos hallados, quedando demostrado que el nivel medio y alto de agotamiento emocional se debe a diversos motivos y que ello podría ser perjudicial para la persona mermando la productividad de las agencias de la institución financiera.

3. Se ha comprobado que los efectos fisiológicos del estrés se relacionan significativamente con el desempeño de los promotores, los resultados estadísticos en esta variable son congruentes con los anteriores, quedando demostrado al obtener el $41 \%$ de promotores que se encuentra influenciados por diversos efectos fisiológicos. De acuerdo a la teoría descrita, se determina que los efectos fisiológicos son cruciales en los trabajadores, constituyéndose en un indicador de mucho cuidado para lograr un desempeño optimo en el trabajador, esto no se refleja en las agencias analizadas, por lo que la conclusión es coherente con la presencia del estrés laboral en los casos identificados.

4. Se concluye que los logros profesionales están directamente relacionados con el desempeño de los promotores, en efecto, el indicador bajo es del 6\% o 3 promotores que no se encuentran de acuerdo con sus logros profesionales. Independientemente de la presencia del estrés laboral, se concluye que los logros deben ser parte de la motivación laboral, pero ello debe tomar un camino adecuado para mejorar la productividad en los promotores y jefes de la institución financiera.

5. Se recomienda realizar un tratamiento médico a los empleados que presentan estrés laboral, y de esta manera proponer medidas necesarias para disminuir este nivel porcentual de personas que presenten síntomas de estrés laboral. Por otro lado, las instituciones financieras deben adoptar medidas clínicas para los promotores que tienen el problema, previniendo que el estrés laboral se convierta en un síndrome tipo burnout. En los casos identificados, se recomienda motivar al personal, a través de una correcta asignación de competencias, una vez logrado la superación del estrés.

6. La recomendación ante la presencia de agotamiento emocional en los promotores de la institución financiera, radica en establecer programas de recreación y confraternidad, que permitan al trabajador sentirse identificado y comprometido con la institución; pero, a la vez el trabajador podrá tomar en cuenta que la empresa valora su esfuerzo y trabajo; quedando comprobado que si las remuneraciones se constituye en el principal factor motivacional, las actividades motivacionales de manera 
intangible podrían incidir en mejorar el ambiente laboral con el objetivo de lograr un menor nivel en los trabajadores.

\section{LISTA DE REFERENCIAS}

Alles, M. (2017) Dirección estratégica de Recursos humanos. Gestión por competencias. Buenos Aires: Granica.

Alles, M. (2005) Desempeño por competencias. Evaluación 360. Buenos Aires: Granica.

Bohlander, G. (2017) Administración de Recursos Humanos. México: Thomson Learning.

Baldoni, J.(may 07,2009). Watch Out for Stress in Your People; [source: Harvard Business Review]. HBR Blog Network. Retrieved from

Borman, W.C. y S.J. Motowidlo (1997): “Task performance and contextual performance: the meaning for personel selection research". Human Performance, $\mathrm{N}^{\circ} 10$, pp. 99-109.

Blum M, Naylor J (2009) Psicología Industrial. Fundamentos teóricos y sociales. México Trillas.

Chiavenato, I. (1993). Administración de Recursos Humanos. Colombia: Mc Graw Hill.

Caligiore, Díaz (2017) Clima organizacional y desempeño de los docentes en la ULA: Estudio de un caso. Recuperado de: http://www.doaj.org/doaj?func=abstract\&id=157984\&toc=y

Campbell, J.P. (2018): “An Overview of the army selection and classification project (Project A)". Personnel Psychology, n43, pp. 231-239.

Campbell, J.P., R.A. McCloy, S.H. Oppler y C. E. Sager (1993): “A theory of performance”. En N. Schmitt y W.C. Borman (eds.), Personnel selection in organization, pp. 35-70. San Francisco: Jossey-Bass.

Cardona, J; Calderón, G. (2018) El impacto del Aprendizaje en el Rendimiento de las Organizaciones. Recuperado de:

http://www.redalyc.org/redalyc/pdf/205/20503202.pdf

Carreño, S.; Medina, E. (2018) Características organizacionales, estrés y consumo de alcohol en trabajadores de una empresa textil mexicana. Recuperado de: http://redalyc.uaemex.mx/pdf/582/58242909.pdf 
Coronado, F. (2018). Factores laborales y niveles estrés laboral en enfermeros de los servicios de áreas críticas y medicina del hospital nacional Daniel A. Carrión. Lima.

Chiavenato I. (2000). Administración proceso administrativo. Tercera edición. Bogotá. McGraw-Hill.

Chiavenato, I. (2002). Gestión del Talento Humano. Primera Edición. México: Editorial McGraw - Hill.

Chiavenato, I. (2007) Administración de Recursos Humanos. El capital humano de las organizaciones. México: McGraw-Hill, Octava Edición.

Conway, J.M. (2018): "Distinguishing contextual performance from task performance for managerial jobs". Journal of Applied Psychology, $\mathrm{N}^{\circ}$ 84, pp. 3-13.

Cruz Marín, Carlos y Vargas Fernández, Luís. (2017). Estrés; entenderlo es manejarlo. México: Alfa Omega.pp.127.

Davis, K., y Newstrom, J. (2018) Comportamiento humano en el trabajo. México: McGraw-Hill, Séptima Edición.

Drucker, P. (2002). Los Desafíos de la Gerencia del Siglo XXI. Bogotá: Grupo Editorial Norma.

Edwards, M. (2018). $360^{\circ}$ Feedback, NY: Amacom, American Management Association. Evaluación $360^{\circ}$. Recuperado de:

http://www.asimetcapacitacion.cl/evaluacion_360grados.htm

Flannery, R.B. \& Wieman, D. (1989). Social support, life stress, and psychological distress: An empirical assessment. Journal of Clinical Psychology, 45(6), 867872.

Flores, Andrea (2010) Tesis presentada en la UNFV, titulada: Síndrome de Burnout y Compromiso Organizacional en personal de una empresa de Telecomunicaciones.

Fontana, David (1992). Control del estrés, Ed. Manual Moderno.

Furnham, A. (1997). Psicología Organizacional y Comportamiento de los individuos en las organizaciones. Oxford University.

Hudtwalcker, R. (2018). Clima Laboral en los equipos deportivos. Revista Digital Buenos Aires, $N^{\circ} 100$ : disponible http://www.efdeportes.com\efd100入clima.htm. 
Gómez González Beatriz y Escobar Alfonso (2002), Neuroanatomía del estrés, en la

Revista Mexicana de Neurociencia, Vol. 3, No. 5, pp. 273-282. Extraído de La Revista Internacional de Psicología.

Gómez González Beatriz y Escobar Alfonso (2002), Neuroanatomía del estrés, en la Revista Mexicana de Neurociencia, Vol. 3, No. 5, pp. 273-282. Extraído de La Revista Internacional de Psicología. http://psicologiarevista.99k.org/El\%20campo\%20de\%20estudio\%20del\%20estre spdf

Gomero Raúl, Palomino Juan, Ruiz Fermín y Yesán Carlos (2005) El Síndrome de Burnout en personal sanitario de los hospitales de la empresa minera de Southern Perú Copper Corporation: estudio Piloto. Revista médica Herediana. Lima. Encontrado en: http://www.scielo.org.pe/scielo.php?pid=S1018130X2005000400002\&script=sci_arttext

Guic S, Bilbao A, Bertin C (2012) Estrés laboral y salud en una muestra de ejecutivos chilenos. Encontrado en: http://www.scielo.cl/scielo.php?pid=S003498872002017000004\&script=sci_arttext

Herzberg (1959) citado por Robbins (2009). The motivation to work. Ney York: John Wiley \& Sons.

Holmes, T., \& Masuda, M. (1972). Psychosomatic syndrome. Psychology Today, April, 71.

Hombrados, I. (1997). Estrés y salud, Ed. Promolibro, pp.616.

Iturral J. (2011). La evaluación del desempeño laboral y su incidencia en los resultados del rendimiento de los trabajadores de la Cooperativa de Ahorro y Crédito OSCUS Ltda. de la ciudad de Ambato en el año 2010. Tesis presentada en la Universidad Técnica de Ambato.

Koontz H. y Weihrich H. (2004). Administración. Una perspectiva global 12a . Edición. México, D.F. McGraw-Hill.

Kessler, R.; Greenberg D. (1981). Linear panel anaíysis: Models of quantitative change. London, UK: Academic Press, Inc. (LONDON) LTD.

Levy-Leboyer, C. (2000). Feedback de 360ª . Barcelona: Gestión 2000. 
Lackritz, J.R. (2004). Exploring burnout among university faculty: incidence, performance, and demographic issues. Teaching and Teacher Education, 20, 713729.

Lee, I. y Wang, H.H. (2002). Perceived occupational stress and related factors in public health nurses. Journal of Nursing Research, 10(4), 253-259.

Leiter, M.P. (1991). The dream denied: professional burnout and the constraints of service organizations. Canadian Psychology, 32, 547-558.

Medina, M.; Armenteros, M.; Guerrero, L.; Barquero, J. (2012) Las Competencias Gerenciales Desde Una Visión Estratégica de las Organizaciones: Un Procedimiento Para Su Identificación y Evaluación del Desempeño (Managerial Skills from a Strategic Viewpoint: A Procedure for Their Identification and Performance Assessment). Recuperado de: http://papers.ssrn.com/sol3/papers.cfm?abstract_id=1953541

Melgosa, Julián (1994). Sin estrés, Ed. Saféliz, pp.189.

Mamani A, Obando R, Uribe A. (2007) Factores que desencadenan el Estrés y sus Consecuencias en el Desempeño Laboral en Emergencia. Encontrado en: http://revistas.concytec.gob.pe/scielo.php?pid=S1816$77132007000100007 \&$ script=sci_arttext

Maslach, C. y Jackson, S.E. (1981). The measurement of experienced burnout. Journal of Occupational Behavior, 2, 99-113.

Motowidlo, S.J. y J.R. Van Scotter (1994): "Evidence that task performance should be distinguished from contextual performance". Journal of Applied Psychology $\mathrm{N}^{\circ}$ 7, pp.475-480.

Otmar, V. (2009). Desempeño de los individuos en las organizaciones de salud de Ecuador. Universidad Técnica Particular de Loja.

Pillow, D.R., Zautra, A.J., \& Sandler, I. (2018). Major life event and minor stressors: Identifying Mediational links in the stress process. Journal of Personality Social Psychology, 70(2), 381-394.

Quiceno J, Vinaccia S (2007) Síndrome de quemarse en el trabajo. Encontrado en: http://portalweb.ucatolica.edu.co/easyWeb2/acta/pdfs/v10n2/art-11_117-125.pdf Rivero, E. (2009). Prevalencia de estrés laboral en trabajadores de salud de la Disa IV Lima Este. Revista Nuevos Paradigmas, 3, 1, 56-65. 
Rodríguez, R. (2011) Los procesos de estrés laboral y desgaste profesional (burnout): diferenciación, actualización y líneas de intervención. Encontrado en: http://scielo.isciii.es/scielo.php?pid=S0465546X2011000500006\&script=sci_arttext

Robbins, S. (2004). Comportamiento Organizacional. Décima Edición. México: Prentice Hall.

Pepper, S.C. (2017) The Sources of Value, Berkeley, University of California Press.

Ponce Ramón, Bulnes Mario, Aliaga Jaime y otros (2005) El síndrome del "quemado" por estrés laboral asistencial en grupos de docentes universitarios. Facultad de Psicología de la UNMSM.

Sherman, B. (2018). Administración de Recursos Humanos, México: Thomson Editores.http://blogs.hbr.org/health-and-well-being/2009/02/new-researchquantifies-the-ef.html

Skerrett, P. (February 19, 2009). New Research Quantifies the Effect of Stress; [source: Harvard Business Review]. HBR Blog Network/ Health and Well-Being. Retrieved from. http://blogs.hbr.org/baldoni/2009/05/watch_out_for_your_people.

Taylor, S.E. (2018). Health Psychology. American Psychologist, 45(1), 40-50.

Varela, O. y E. Salgado (2009). El Desempeño de los individuos en las organizaciones", pp. 23-45.

Wheeler, R.J. \& Frank, M.A. (1988). Identification of stress buffers. Behavioral Medicine, 14(2), 78-89.

Weiten, W. (1988). Pressure as a form of stress and its relationship to psychological symptomatology. Journal of Social Clinical Psychology, 6(1), 127-139.

Zaldívar, Dionisio (2018). Conocimiento y dominio del estrés. Ed. Científico - técnico, p.111. 